\title{
A retrospective study on intensity-modulated radiation therapy combined with chemotherapy after D2 radical surgery for gastric carcinoma
}

\author{
WENGUANG LUO, HONGYAN ZHANG, YUFEI ZHAO, LIN WANG, \\ LIJUN QI, JINGJING RAN, LEI LIU and AIDONG WU \\ Department of Radiation Oncology, Anhui Provincial Hospital, Hefei, Anhui 230001, P.R. China
}

Received September 23, 2015; Accepted February 22, 2016

DOI: $10.3892 / \mathrm{mco} .2016 .804$

\begin{abstract}
In order to investigate the clinical value of different chemotherapies, the efficacy of intensity-modulated radiation therapy with concurrent chemotherapy following D2 radical surgery for gastric carcinoma was evaluated in this study. A total of 102 patients who underwent D2 radical surgery for gastric carcinoma followed by concurrent chemoradiotherapy (CRT) between January, 2008 and March, 2012, were selected. The 5/7 field intensity-modulated radiation therapy was used, with a planning target volume dose of $45 \mathrm{~Gy}$ in 25 fractions over 5 weeks. Among these patients, 45 were administered $400 \mathrm{mg} / \mathrm{m}^{2} /$ day fluorouracil and $20 \mathrm{mg} / \mathrm{m}^{2} /$ day tetrahydrofurfuryl alcohol through intravenous infusion 4 days before and 3 days after the radiotherapy (F-CRT group), while 57 patients received $825 \mathrm{mg} / \mathrm{m}^{2}$ capecitabine orally twice a day (C-CRT group). The 3-year overall and the disease-free survival rates were 75.5 and $70.5 \%$, respectively. The overall 3-year survival rates of the F-CRT and C-CRT groups were 72.2 and $78.5 \%$ $(\mathrm{P}>0.05)$, respectively, and the 3-year disease-free survival rates were 67.7 and $72.8 \%(\mathrm{P}>0.05)$, respectively. No significant differences were observed between the two groups. However, during the concurrent CRT, significant differences were found in the incidence of grade 1-2 haematological toxicity between the F-CRT and C-CRT groups (73.3 vs. 50.9\%, respectively; $\left.\chi^{2}=5.320, \mathrm{P}=0.021\right)$. Significant differences were also found in the incidence of grade 1-2 gastrointestinal reactions between the two groups (77.8 vs. $57.9 \%$, respectively; $\chi^{2}=4.474$, $\mathrm{P}=0.034)$. Therefore, intensity-modulated radiation therapy combined with concurrent chemotherapy following D2 radical surgery for gastric cancer was found to be safe and effective.
\end{abstract}

Correspondence to: Dr Wenguang Luo or Professor Hongyan Zhang, Department of Radiation Oncology, Anhui Provincial Hospital, 17 Rujiang Road, Hefei, Anhui 230001, P.R. China

E-mail: 13965096716@163.com

E-mail: zhanghongyan3334@qq.com

Key words: gastric carcinoma, intensity-modulated radiation therapy, chemotherapy, D2 surgery, oral capecitabine therapy, intravenous fluorouracil therapy
In addition, radiotherapy was better tolerated and more likely to be completed using C-CRT rather than F-CRT.

\section{Introduction}

Gastric carcinoma, a common malignant tumor of the digestive tract, is the fourth more common cause of morbidity and the second cause of mortality among all malignant tumors worldwide, and is particularly common in Latin America, Eastern Europe and East Asian countries $(1,2)$. The radical therapy of gastric carcinoma mainly relies on surgical resection; however, local recurrence and distant metastasis may still occur, even in patients who have undergone complete surgical resection (3-5). Among patients with failed surgical intervention, 90\% experienced local recurrence, which led to death in $\sim 80 \%$ of these patients, particularly those with serosal invasion or lymphatic metastasis $(6,7)$. The prognosis with surgery alone is poor for patients with locally advanced gastric carcinoma, while the 5 -year survival rate is only $\sim 8-34 \%$ (8). In order to improve the survival rate of patients with gastric carcinoma and reduce the recurrence rate, adjuvant therapy following gastrectomy has become a consensus (9). However, chemotherapy alone after surgery did not confer an obvious survival benefit to such patients in the majority of studies $(9,10)$.

Since the results of the INT-0116 study (11) were published in 2001, adjuvant concurrent chemoradiotherapy (CRT) after surgery has been commonly used by oncologists for the treatment of gastric carcinoma; therefore, certain European and American guidelines have included adjuvant CRT and recommend it as the standard treatment following surgery. Among selected patients, the proportion of those receiving limited lymph node dissection (D0 or D1) is $\leq 90 \%$, whereas the proportion of those receiving extended D2 lymph node dissection (D2) is only $10 \%$ (11). However, in some Asian countries, patients with gastric carcinoma are more likely to receive D2 radical surgery, which limits the significance of this study for Asian patients. Thus, Kim et al compared patients who had received adjuvant concurrent CRT following D2 radical surgery for gastric carcinoma with those who had received surgery alone, and demonstrated that adjuvant concurrent CRT following D2 radical surgery may improve the survival rate and reduce the recurrence rate (12). The ARTIST trial further compared patients who had received concurrent 
CRT following D2 surgery for gastric carcinoma with those who had received chemotherapy alone, and demonstrated that the overall 3-year disease-free survival rate was marginally higher compared with that of the group with concurrent CRT, although the difference was not statistically significant (13).

Intensity-modulated radiation therapy (IMRT) is a more advanced radiotherapy technique, which is able to concentrate high doses of radiation on the target area, while better protecting the adjacent normal tissue and has a dose advantage compared with conventional radiotherapy and three-dimensional conformal radiation therapy (3DCRT) following surgery for gastric carcinoma $(14,15)$.

To evaluate the efficacy of IMRT combined with concurrent chemotherapy following D2 radical surgery for gastric carcinoma and investigate the clinical value of its combination with different chemotherapies, we conducted a study including 102 patients who had received IMRT combined with concurrent chemotherapy with intravenous infusion of fluorouracil (F-CRT) or oral capecitabine (C-CRT) following D2 radical surgery for gastric carcinoma.

\section{Patients and methods}

Patient eligibility. A total of 102 patients treated with D2 radical surgery for gastric carcinoma were enrolled from January, 2008 to March, 2012. The characteristics of the patients are shown in Table I. The criteria for inclusion were as follows: Pathologically proven diagnosis of gastric adenocarcinoma; radical surgical resection (R0) of the tumor $\leq 4$ weeks prior; D2 lymph node dissection; age $\geq 18$ and $\leq 70$ years; World Health Organization performance status score $\leq 2$; TNM stage T3, T4 or N+ (IB-III) according to the American Joint Committee on Cancer (AJCC), 7th edition (16); normal cardiac, hepatic and renal function; normal bone marrow function (neutrophil count $\geq 1,500 / \mu 1$, blood platelet $100,000 / \mu 1$, hemoglobin level $\geq 10 \mathrm{~g} / \mathrm{dl}$ ); no malignant tumor at other sites; no intolerance of CRT due to various systemic diseases; no residual tumor or positive surgical margins after surgery; no TNM stage T1-2N0; no distant metastasis (M1); and no D0/D1 lymph node dissection. The present study was approved by the Ethics Committee of Anhui Provincial Hospital (Hefei, China) and all participating patients provided written informed consent.

Radiotherapy. Radiotherapy was initiated within 4 weeks after surgery. All the patients were required to fast for at least $4 \mathrm{~h}$, and were scanned after orally taking $20 \mathrm{ml}$ compound diatrizoate meglumine solution and $500-800 \mathrm{ml}$ water to highlight the gastric stump and part of the intestinal loop structure. The patients were maintained at the supine position by a restraining device to undergo computed tomography (CT) enhancement scanning, which required a 5-mm stratum depth. The scanning range included the section between the fourth thoracic vertebra and the fourth lumbar vertebra, and the images were transferred to a treatment planning system after restructuring as $2.5 \mathrm{~mm}$ per stratum. First, the clinical target volume (CTV), which includes the preoperative tumor bed, anastomotic stoma, duodenal stump and high-risk lymphatic drainage area, was delineated on the restructured CT images by combining contrast agent development, postoperative silver clips and preoperative imaging data $(17,18)$. Planning target volume (PTV) included
CTV taking into consideration organ excursion, with the enlarged irradiation area caused by repeatability error of the patient's position during positioning and treatment, as well as taking into account a change of target positions and target volumes to ensure the irradiation dose of CTV. Accordingly, PTV usually included a 7-10-mm margin around CTV, with a $10-\mathrm{mm}$ craniocaudal margin. The organs at risk included the spinal cord, liver, heart, kidney and small intestine.

The number of the beams and incidence angle were adjusted according to the ray path and the association of the tumor target volume with surrounding normal tissue, while the dose of each beam was weighted with the limits of the organs at risk calculated. The treatment plan was formulated through automatic optimization by a computer reverse planning system. Isocenter irradiation was conducted using 5 or 7 intensity-modulated irradiation fields, which were coplanar and non-through. The 5-field IMRT beam orientation was usually at the following gantry angle: $0,72,144,216$ and $288^{\circ}$; the 7-field IMRT beam orientation was usually at the following gantry angle: $0,51,103$, $154,206,257$ and $309^{\circ}$. Based on the patient's target shape and the dose to target volume and normal tissue, adjustments were made until the plan requirements were met. The prescription dose of $95 \%$ PTV was 45 Gy in 25 fractions (1.8 Gy/fr). The doses to each organ at risk were as follows: Maximum dose to the spinal cord $\leq 45 \mathrm{~Gy}$; liver V30<30\%; mean dose to each kidney <18 Gy, V15 $\leq 50 \%$; small intestine V50 $\leq 10 \%$; heart dose $\mathrm{V} 40 \leq 30 \%$, V30 $\leq 40 \%$; whole-lung dose V $20 \leq 25 \%$, V5 $\leq 60 \%$.

Chemotherapy. For comparison, during concurrent CRT the patients were separated into two groups: 45 patients received intravenous infusion of $400 \mathrm{mg} / \mathrm{m}^{2} /$ day 5 -fluorouracil and $20 \mathrm{mg} / \mathrm{m}^{2} /$ day tetrahydrofolic acid (THFA) 4 days before and 3 days after radiotherapy; and 57 patients received $825 \mathrm{mg} / \mathrm{m}^{2}$ oral capecitabine twice each day (morning and evening) during radiotherapy, and four cycles of XELOX chemotherapy (capecitabine $1,000 \mathrm{mg} / \mathrm{m}^{2}$ on days $1-14+$ oxaliplatin $130 \mathrm{mg} / \mathrm{m}^{2}$ on day 1 , repeated every 3 weeks) 4 weeks after the end of radiotherapy.

Evaluation of toxicity. The Common Terminology Criteria for Adverse Events v3.0 (19) of the National Cancer Institute were used for the evaluation of toxicity.

Statistical analysis. Statistical analysis was performed using SPSS 19.0 software (Armonk, NY, USA). The Chi-square test was used for the comparison of rates and the Student's t-test was used for the comparison of means. The survival rate was calculated by non-parametric Kaplan-Meier test, while the log-rank test was used to compare survival curve distribution between the two groups and to determine whether the difference was statistically significant. The multivariate Cox regression model was used to perform multivariate analysis of prognosis. $\mathrm{P}<0.05$ was considered to indicate statistically significant differences.

\section{Results}

Patients. A total of 102 patients who met the aforementioned criteria were enrolled in this study. The characteristics of these patients are listed in Table I. There were no significant 
Table I. Clinicopathological characteristics of the 102 patients.

\begin{tabular}{|c|c|c|c|}
\hline Characteristics & $\begin{array}{l}\text { Total number } \\
\quad(n=102)\end{array}$ & $\begin{array}{c}\text { F-CRT, n }(\%) \\
(n=45)\end{array}$ & $\begin{array}{c}\text { C-CRT, n (\%) } \\
(\mathrm{n}=57)\end{array}$ \\
\hline \multicolumn{4}{|l|}{ Gender } \\
\hline Male & 69 & $31(68.9)$ & $38(66.7)$ \\
\hline Female & 33 & $14(31.1)$ & $19(33.3)$ \\
\hline \multicolumn{4}{|l|}{ Age, years } \\
\hline$\leq 55$ & 56 & $24(53.3)$ & $32(56.1)$ \\
\hline$>55$ & 46 & $21(46.7)$ & $25(43.9)$ \\
\hline \multicolumn{4}{|l|}{ Tumor site } \\
\hline Esophagogastric junction & 22 & $9(20.0)$ & $13(22.8)$ \\
\hline Gastric body & 24 & $11(24.4)$ & $13(22.8)$ \\
\hline Gastric antrum & 56 & $25(55.6)$ & $31(54.4)$ \\
\hline \multicolumn{4}{|l|}{ Pathological type } \\
\hline Canalicular and papillary adenocarcinoma & 62 & $27(60.0)$ & $35(61.4)$ \\
\hline Poorly differentiated adenocarcinoma & 21 & $10(22.2)$ & $11(19.3)$ \\
\hline Others & 19 & $8(17.8)$ & $11(19.3)$ \\
\hline \multicolumn{4}{|l|}{ Lymph node metastasis } \\
\hline Yes & 66 & $30(66.7)$ & $36(63.2)$ \\
\hline No & 36 & $15(33.3)$ & $19(36.8)$ \\
\hline \multicolumn{4}{|l|}{ TNM stage } \\
\hline IB & 11 & $5(11.1)$ & $6(10.5)$ \\
\hline II & 38 & $17(37.8)$ & $21(36.8)$ \\
\hline III & 53 & $23(51.1)$ & $30(52.7)$ \\
\hline \multicolumn{4}{|l|}{ Vascular cancer embolus or neural invasion } \\
\hline Positive & 25 & $11(24.4)$ & $14(24.6)$ \\
\hline Negative & 77 & $34(75.6)$ & $43(75.4)$ \\
\hline
\end{tabular}

F-CRT, concurrent chemoradiotherapy with intravenous infusion of fluorouracil; C-CRT, concurrent chemoradiotherapy with oral capecitabine.

differences in gender distribution, age, tumor site, pathological type, TNM stage, or the extent of neurovascular invasion between the F-CRT and C-CRT groups. Overall, 98 patients completed the concurrent CRT; 4 patients only completed radiotherapy but not chemotherapy, due to the toxic side effects, including 3 patients from the F-CRT group who only completed the first 4-day concurrent chemotherapy without the subsequent 3-day chemotherapy, and 1 patient from the C-CRT group who received concurrent capecitabine for only 2 weeks. A total of 6 patients did not complete the subsequent 4-cycle XELOX chemotherapy, of whom 4 patients were from the F-CRT group (2 patients only received two cycles of chemotherapy, 1 received only one cycle, and 1 patient did not receive any subsequent chemotherapy) and 2 patients were from the C-CRT group (1 received only two cycles of chemotherapy, and 1 did not receive any subsequent chemotherapy).

Safety evaluation. The individual toxic side effects in the F-CRT and C-CRT groups during the entire course of treatment are shown in Table II. Overall, the incidence of grade 3 hematological toxicities (leukopenia, anemia and thrombocytopenia) was $23.5 \%$; grade 3 gastrointestinal reactions (nausea, vomiting and diarrhea) $13.7 \%$; grade 1-2 hepatic and renal function impairment $9.8 \%$; and grade $\geq 3$ hepatic and renal function impairment $0 \%$. The most common grade 1-2 hema- tological toxicity was anemia (76.5\%), and the most common grade 3 toxicity was leukopenia (21.6\%). Grade 1-2 gastrointestinal reactions included nausea (71.6\%), vomiting $(34.3 \%)$ and diarrhea $(26.5 \%)$, and grade 3 gastrointestinal reactions included nausea (10.8\%), vomiting (3\%) and diarrhea (1\%). During the entire course of treatment, the incidence of grade 3 hematological toxicities (leukopenia, anemia and thrombocytopenia) in the F-CRT and C-CRT groups was 28.9 and $19.3 \%$, respectively, and the incidence of grade 3 gastrointestinal reactions (nausea, vomiting and diarrhea) was 17.8 and $10.5 \%$, respectively. Grade 3 hematological toxicities and gastrointestinal reactions were more common in the F-CRT group, but the difference was not statistically significant. The incidence of grade 1-2 hepatic and renal function impairment in the F-CRT and C-CRT groups was 11.1 and 8.8\%, respectively, but the difference was again not statistically significant. The incidence of grade 1-2 hand-foot syndrome was 35.6 and $40.4 \%$, respectively, with 1 patient in each group experiencing grade 3 hand-foot syndrome, without statistically significant differences between the two groups (Table II).

As shown in Table III, during the concurrent CRT, the incidence of grade 1-2 hematological toxicities in the F-CRT group was significantly higher compared with that in the C-CRT group (73.3 and $50.9 \%$, respectively; $\mathrm{P}=0.021)$. The incidence of grade 1-2 gastrointestinal reactions in the F-CRT group was 
Table II. Toxicities in the F-CRT and C-CRT groups during the entire course of treatment.

\begin{tabular}{|c|c|c|c|c|}
\hline \multirow[b]{2}{*}{ Toxicities } & \multicolumn{2}{|c|}{ F-CRT $(n=45)$} & \multicolumn{2}{|c|}{ C-CRT $(n=57)$} \\
\hline & $\begin{array}{c}\text { Grade } 1-2 \\
\mathrm{n}(\%)\end{array}$ & $\begin{array}{c}\text { Grade } 3-4 \\
\mathrm{n}(\%)\end{array}$ & $\begin{array}{c}\text { Grade } 1-2 \\
\mathrm{n}(\%)\end{array}$ & $\begin{array}{c}\text { Grade } 3-4 \\
\mathrm{n}(\%)\end{array}$ \\
\hline Nausea & $34(75.6)$ & $6(13.3)$ & $39(68.4)$ & $5(8.8)$ \\
\hline Vomiting & $17(37.8)$ & $2(4.4)$ & $18(31.2)$ & $1(1.8)$ \\
\hline Diarrhea & $12(26.7)$ & $1(2.2)$ & $15(26.3)$ & $0(0.0)$ \\
\hline Leukopenia & $28(62.2)$ & $11(24.4)$ & $34(59.6)$ & $10(17.5)$ \\
\hline Anemia & $35(77.8)$ & $2(4.4)$ & $43(75.4)$ & $2(3.6)$ \\
\hline Thrombocytopenia & $13(28.9)$ & $1(2.2)$ & $17(29.8)$ & $0(0.0)$ \\
\hline HFS & $16(35.6)$ & $1(2.2)$ & $23(40.4)$ & $1(1.8)$ \\
\hline ALT & $4(8.9)$ & $0(0.0)$ & $5(8.8)$ & $0(0.0)$ \\
\hline GFR & $1(2.2)$ & $0(0.0)$ & $0(0.0)$ & $0(0.0)$ \\
\hline
\end{tabular}

F-CRT, concurrent chemoradiotherapy with intravenous infusion of fluorouracil; C-CRT, concurrent chemoradiotherapy with oral capecitabine; HFS, hand-foot syndrome; ALT, alanine aminotransferase; GFR, glomerular filtration rate.

Table III. Toxicities in the F-CRT and C-CRT groups during the concurrent radiochemotherapy.

\begin{tabular}{lcccc}
\hline & \multicolumn{2}{c}{ F-CRT $(\mathrm{n}=45)$} & & C-CRT $(\mathrm{n}=57)$ \\
\cline { 2 - 5 } Toxicities & $\begin{array}{c}\text { Grade 1-2 } \\
\mathrm{n}(\%)\end{array}$ & $\begin{array}{c}\text { Grade 3-4 } \\
\mathrm{n}(\%)\end{array}$ & $\begin{array}{c}\text { Grade 1-2 } \\
\mathrm{n}(\%)\end{array}$ \\
\hline Gastrointestinal & $35(77.8)$ & $1(2.2)$ & $33(57.9)$ & 0 \\
Hematological & $33(73.3)$ & $1(2.2)$ & $29(50.9)$ & $1(1.8)$ \\
\hline
\end{tabular}

F-CRT, concurrent chemoradiotherapy with intravenous infusion of fluorouracil; C-CRT, concurrent chemoradiotherapy with oral capecitabine.
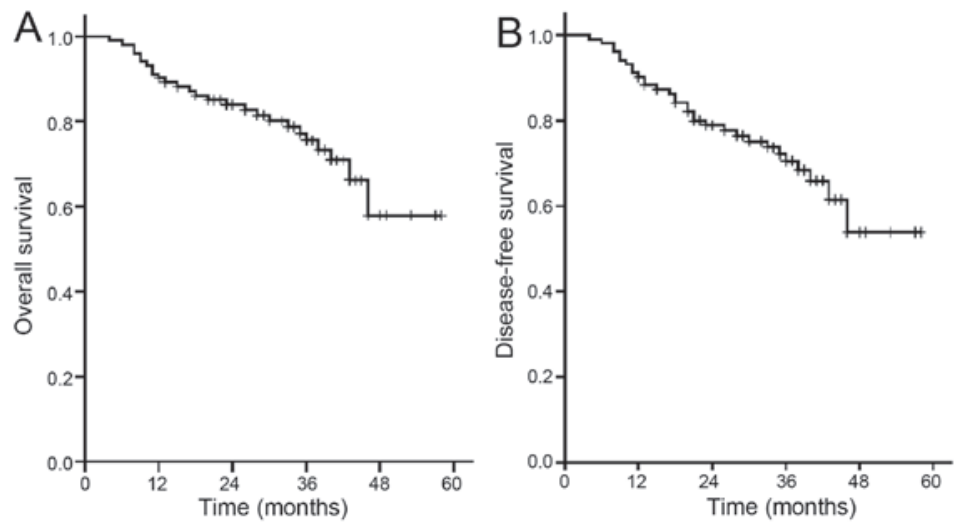

Figure 1. (A) Overall survival rate and (B) disease-free suvival rate of the 102 gastric cancer patients in this study.

also significantly higher compared with that in the C-CRT group (77.8 and $57.9 \%$, respectively; $\mathrm{P}=0.034)$. However, during the concurrent CRT, no statistically significant difference was observed in grade 3-4 hematological toxicities and gastrointestinal reactions between the F-CRT and the C-CRT groups.

Survival analysis. The median follow-up time in the overall population was 40 months (range, 18-61.2 months), the follow-up rate was $98 \%$, and loss to follow-up was considered as death. The 3-year survival rate was $75.5 \%$ and the disease-free survival rate was $70.5 \%$ (Fig. 1), while the survival rate was $84.3 \%$ in a local group.

As shown in Fig. 2, the total 3-year survival rate of the F-CRT and C-CRT groups was 72.2 and 78.5\%, respectively $(\mathrm{P}>0.05)$, and the 3-year disease-free survival rate was 67.7 and $72.8 \%$, respectively $(\mathrm{P}>0.05)$. No statistically significant differences were observed. 
Table IV. Prognostic univariate analysis in the overall population.

\begin{tabular}{|c|c|c|c|c|}
\hline Variables & Number & 3 -year survival rate & $\chi^{2}$ & P-value \\
\hline \multicolumn{5}{|l|}{ Gender } \\
\hline Male & 69 & 76.8 & 2.613 & 0.116 \\
\hline Female & 33 & 72.7 & & \\
\hline \multicolumn{5}{|l|}{ Age, years } \\
\hline$\leq 55$ & 56 & 73.2 & 1.436 & 0.217 \\
\hline$>55$ & 46 & 78.3 & & \\
\hline \multicolumn{5}{|l|}{ Tumor site } \\
\hline Esophagogastric junction & 22 & 68.2 & 5.129 & 0.078 \\
\hline Gastric body & 24 & 75.0 & & \\
\hline Gastric antrum & 56 & 78.6 & & \\
\hline \multicolumn{5}{|l|}{ Pathological type } \\
\hline $\begin{array}{l}\text { Canalicular and papillary } \\
\text { adenocarcinoma }\end{array}$ & 62 & 79.0 & 5.682 & 0.062 \\
\hline Poorly differentiated & 21 & 71.4 & & \\
\hline adenocarcinoma & 19 & 68.4 & & \\
\hline \multicolumn{5}{|l|}{ Others } \\
\hline \multicolumn{5}{|l|}{ Lymph node metastasis } \\
\hline Yes & 66 & 63.8 & 8.055 & 0.005 \\
\hline No & 36 & 84.4 & & \\
\hline \multicolumn{5}{|l|}{ TNM stage } \\
\hline IB & 11 & 90.9 & 7.420 & 0.024 \\
\hline II & 38 & 86.8 & & \\
\hline III & 53 & 64.2 & & \\
\hline \multicolumn{5}{|l|}{$\begin{array}{l}\text { Vascular cancer embolus } \\
\text { or neural invasion }\end{array}$} \\
\hline Positive & 25 & 70.2 & 4.426 & 0.038 \\
\hline Negative & 77 & 82.4 & & \\
\hline \multicolumn{5}{|l|}{ Chemotherapy } \\
\hline $5-\mathrm{FU}+\mathrm{CF}$ & 45 & 72.2 & 3.204 & 0.085 \\
\hline Capecitabine & 57 & 78.5 & & \\
\hline
\end{tabular}

5-FU, 5-fluorouracil; CF, leucovorin.

Analysis of prognostic factors. Lymph node metastasis, TNM stage, vascular cancer embolus and neural invasion are usually the factors that affect survival. In our study, as shown in Table IV, the 3-year survival rate of patients with and without lymph node metastasis was 63.8 vs. $84.4 \%$, respectively $(\mathrm{P}=0.005$; Fig. 3). The 3-year survival rate of patients with stage IB, II and III disease was 90.9, 86.8 and $64.2 \%$, respectively ( $\mathrm{P}=0.024$; Fig. 4$)$. The 3 -year survival rate of patients with positive and negative neurovascular invasion was 70.2 and $82.4 \%$, respectively $(\mathrm{P}=0.038$; Fig. 5). In accordance with previous studies $(11,12)$, lymph node metastasis, TNM stage and vascular cancer embolus or neural invasion are statistically significant prognostic factors, while factors such as gender, age, tumor site, pathological type and different chemotherapies were not significantly associated with prognosis.

To determine the independent prognostic factors, multivariate Cox regression analysis was used. As shown in Table V,

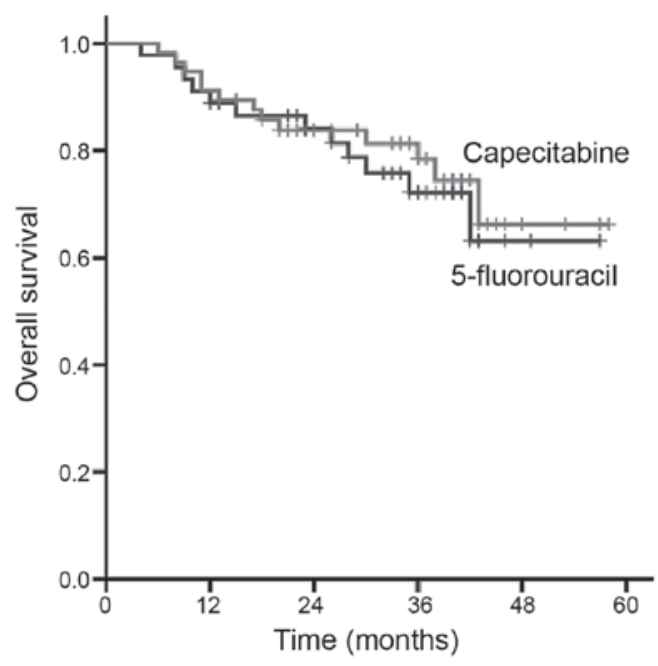

Figure 2. Overall survival rate with the two different chemotherapies. 
Table V. Multivariate Cox regression analysis of prognosis after surgery for gastric cancer.

\begin{tabular}{lccccc}
\hline Variables & $\begin{array}{c}\text { Regression } \\
\text { coefficient (B) }\end{array}$ & $\begin{array}{c}\text { Standard } \\
\text { error }\end{array}$ & $\begin{array}{c}\text { Statistics } \\
\text { (Wald) value }\end{array}$ & P-value & $\begin{array}{c}95 \% \text { confidence } \\
\text { interval }\end{array}$ \\
\hline TNM stage & 0.304 & 0.121 & 5.136 & 0.032 & $1.084-1.843$ \\
Lymph node metastasis & 0.362 & 0.146 & 6.523 & 0.010 & $1.097-1.965$ \\
Vascular/nerve invasion & 0.148 & 0.092 & 2.385 & 0.138 & $0.984-1.764$ \\
\hline
\end{tabular}

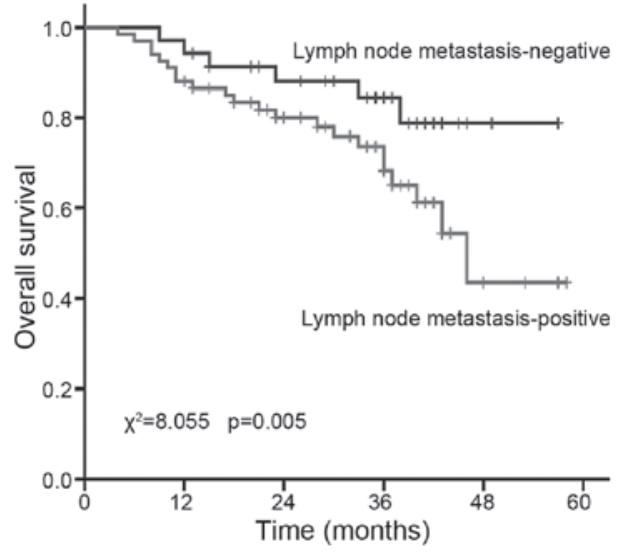

Figure 3. Effect of lymph node status on overall survival rate.

lymph node metastasis and TNM stage were independent prognostic factors, while vascular and/or neural invasion was not identified as an independent prognostic factor.

\section{Discussion}

The majority of previous studies have compared adjuvant concurrent CRT after surgery with surgery alone in patients with gastric carcinoma undergoing D0/D1 surgery $(11,20,21)$, while only a limited number of studies compared CRT after D2 radical surgery with surgery alone (12). As D2 resection (surgery with an enlarged range of lymph node dissection) is common in Asia, particularly in Eastern Asia, this study was focused on such patients in order to estimate the effects of concurrent CRT following D2 resection in gastric carcinoma.

The clinical SWOG 9008/INT-0116 study (11) was a milestone study designed to observe the effects of surgery and postoperative concurrent CRT in patients with resectable gastric and gastroesophageal junction carcinoma. The study included two groups, the R0 resection with concurrent CRT group (281 patients) and the surgery alone group (275 patients). All the patients had stage IB-IV (M0) gastric or gastroesophageal junction carcinoma, of whom $68 \%$ had stage $\mathrm{T} 3$ or T4 lesions, and $85 \%$ had lymph node metastasis (11). In the concurrent CRT group, the local recurrence rate was significantly reduced compared with that in the surgery alone group (19 vs. $29 \%$, respectively), the median survival was significantly prolonged (36 vs. 27 months, respectively), the 3-year disease-free rate was significantly improved (48 vs. $31 \%$, respectively), the survival rate was significantly improved (50 vs. $41 \%$, respectively; $\mathrm{P}=0.005)$ and the incidence of grade 3-4 adverse reactions was 41 and $32 \%$, respectively (11). Moreover, concurrent CRT

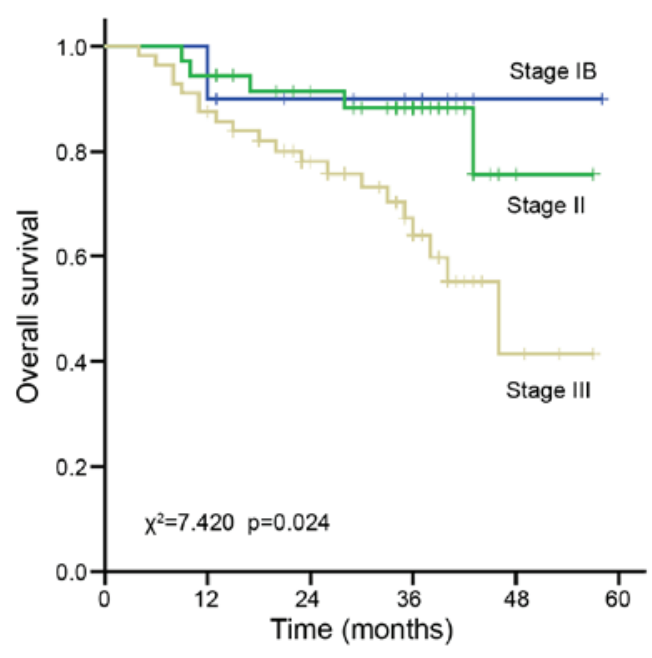

Figure 4. Effect of TNM stage on overall survival rate.

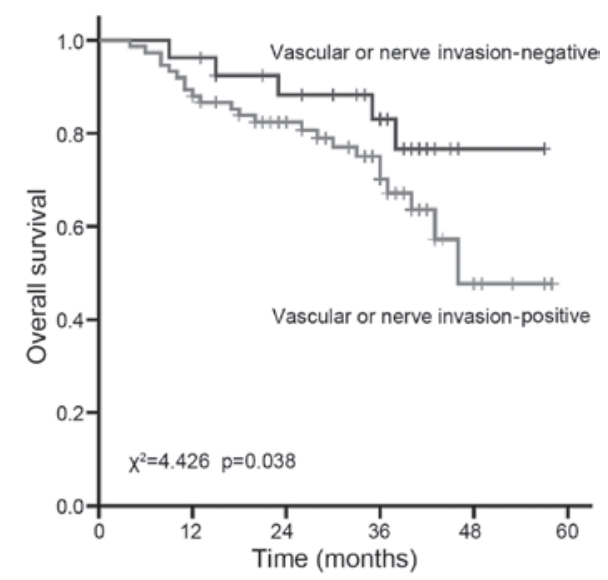

Figure 5. Effect of vascular cancer embolus and neural invasion status on overall survival rate.

after surgery was shown to benefit patients with stage IB-IV (M0) gastric cancer without increasing chronic toxicity in a follow-up study (20). The INT-0116 study established the use of concurrent CRT as standard postoperative adjuvant treatment of gastric carcinoma in the US and several European countries (21). However, a major disadvantage of that study was that only $10 \%$ of the selected patients received D2 resection, while $36 \%$ of the patients received D1 resection, and over half of the patients $(54 \%)$ received $<\mathrm{D} 0$ resection.

Kim et al compared the outcome of gastric carcinoma patients with or without CRT following D2 radical surgery (12), of whom 554 patients had CRT after D2 radical 
surgery, while 446 patients had D2 radical surgery alone. A significant increase in the median survival time and median relapse-free survival time were observed in the concurrent CRT group compared with the surgery alone group. The 5-year survival rate $(57.1$ vs. $51.0 \%, \mathrm{P}=0.0198)$ and relapse-free rate (54.5 vs. $4.9 \%, \mathrm{P}=0.0161$ ) of the concurrent CRT group were significantly higher compared with those in the surgery alone group, and the mortality risk was reduced by $20 \%$ (12). These data indicated that concurrent CRT following D2 gastric cancer surgery may improve the survival rate and reduce the recurrence rate.

In the ARTIST study (13), which aimed to compare the results of the gastric cancer patients who had received concurrent CRT with those of patients who had received chemotherapy alone, 228 patients were randomized into the 6-cycle adjuvant chemotherapy XP group (capecitabine plus cisplatin) and 230 patients were randomized to receive 2-cycle concurrent XP chemotherapy, sequential concurrent CRT (total dose of 45 Gy in 25 fractions + capecitabine), and sequential 2-cycle XP chemotherapy. After a median follow-up of 53.2 months, no significant differences were found in 3-year disease-free survival rate $(78.2 \%$ for CRT vs. $74.2 \%$ for chemotherapy; $\mathrm{P}=0.0862$ ), but only marginal differences were found in the 3 -year disease-free survival rate in patients with positive lymph nodes (77.5 vs. $72.3 \%$, respectively; $\mathrm{P}=0.0365$ ) (13).

In our study, the 3 -year survival rate in patients who received concurrent CRT following gastric cancer surgery was $75.5 \%$, and the disease-free survival rate was $70.5 \%$, while the local control rate was $84.3 \%$. However, a limitation of our study was that we only investigated concurrent CRT after surgery, without setting up control groups to compare with surgery alone and postoperative chemotherapy alone. However, the 3-year disease-free survival rate was higher compared with that reported in the INT-0116 study, which may be explained by the fact that all our patients had received D2 radical surgery, suggesting a better prognosis with chemotherapy after D2 surgery rather than D0/D1 surgery in terms of disease-free survival. When compared to the ARTIST study, our study demonstrated a lower 3-year disease-free survival rate, possibly because all our patients were at advanced TNM stage, which is also the cause for the outcome differences with treatments between European patients and patients from Japan and Korea.

Since the adjuvant concurrent CRT was limited by the tolerance dose of major organs, such as the gastrointestinal tract, liver and kidney, the conventional radiotherapy usually led to the intolerable radiation reactions in the gastrointestinal tract, liver and other organs at risk. Compared with conventional radiotherapy and 3DCRT, IMRT had the advantage of three-dimensional conformity of high doses to the target volume, increasing the total dose delivered $(14,15)$. Milano et al (14) also reported that the IMRT reduced the liver V30 (63.6 vs. $18.9 \%, \mathrm{P}=0.010$ ) and right kidney V20 (20.9 vs. $11.6 \%, \mathrm{P}=0.027$ ) compared with $2 / 3$-field 3 DCRT. Boda-Heggemann et al (22) observed that the V30 of the left and right kidneys was 26.8 vs. $19.5 \%$, respectively $(\mathrm{P}=0.0015)$ when investigating 27 patients who received concurrent CRT with 3DCRT. A study conducted by Stanford University (23) also indicated that IMRT led to a reduction of the liver V30 from 28 to $16.1 \%$ compared with 3DCRT. Moreover, IMRT also improved the postoperative gastric cancer treatment. Badakhshi et al (24) investigated 25 cases of patients who received concurrent CRT with IMRT after gastric cancer surgery, and observed that the incidences of grade 3 nausea and diarrhea during treatment were 4 and $8 \%$, respectively, and the incidences of grade 3 decrease in hemoglobin level, leukocyte and platelet counts were 12, 25 and 4\%, respectively.

Both the INT-0116 study and ARTIST trial used front-back parallel opposed-field radiotherapy (11). In our study, we used 5/7-field IMRT, with an incidence of grade 3 hematological toxicities during treatment of $23.5 \%$; grade 3 gastrointestinal reactions of $13.7 \%$; grade $1-2$ liver and kidney function impairment of $9.8 \%$; and grade 3 liver and kidney function impairment of $0 \%$; these findings were similar to the results reported by Badakhshi et al (24). In addition, we observed that grade 3 hematological toxicities and gastrointestinal reactions mostly occurred due to the cumulative effect of subsequent adjuvant chemotherapy, and the toxic effects were usually grade 1-2 during concurrent CRT. Moreover, all the patients were able to tolerate concurrent CRT after symptomatic treatment.

The concurrent chemotherapy used by the INT-0116 study was 5-fluorouracil + leucovorin, and the efficacy and safety of oral capecitabine as concurrent CRT following gastric cancer surgery had also been demonstrated (25-27). The CLASSIC trial (28) demonstrated the effectiveness and good tolerance of capecitabine plus oxaliplatin as adjuvant therapy after gastric cancer surgery. To determine the differences between 5-fluorouracil and capecitabine, we compared the F-CRT and C-CRT groups following concurrent CRT, with 45 patients receiving concurrent chemotherapy for the first 4 and last 3 days of radiotherapy with $400 \mathrm{mg} / \mathrm{m}^{2} /$ day fluorouracil and $20 \mathrm{mg} / \mathrm{m}^{2} /$ day THFA, and 57 patients receiving $825 \mathrm{mg} / \mathrm{m}^{2} /$ day capecitabine twice daily (in the morning and evening) during the radiotherapy. The 3-year overall survival rate of the F-CRT and C-CRT groups was 72.2 and $78.5 \%$, respectively ( $P>0.05)$; and the 3-year disease-free survival rate was 67.7 and $72.8 \%$, respectively $(P>0.05)$; the differences were not statistically significant. During the entire therapy course, the grade 1-2 hematological toxicities and grade 1-2 gastrointestinal reactions were similar between the F-CRT and C-CRT groups; the incidence of grade 3 hematological toxicities was 28.9 and $19.3 \%$, respectively; the incidence of grade 3 gastrointestinal reactions was 17.8 and $10.5 \%$, respectively; there was no statistically significant difference. However, the incidences of grade 1-2 hematological toxicities in the F-CRT and C-CRT groups were 73.3 vs. $50.9 \%$, respectively $\left(\chi^{2}=5.320 ; \mathrm{P}=0.021\right)$ and the incidences of grade 1-2 gastrointestinal reactions 77.8 vs. $57.9 \%$, respectively $\left(\chi^{2}=4.474 ; \mathrm{P}=0.034\right)$ during concurrent CRT. Thus, concurrent chemotherapy with capecitabine reduced grade 1-2 hematological toxicities and grade 1-2 gastrointestinal reactions in the C-CRT group, and was able to help the patients tolerate and complete the radiotherapy.

As regards prognostic factors following gastric cancer surgery, most scholars consider the status of lymph node metastasis to be an important independent factor affecting the prognosis of gastric cancer $(29,30)$. Other studies demonstrated that the prognosis of the patients was associated with the absolute number of positive lymph nodes when the lymph node sampling was sufficient (16 required by TNM, AJCC 7th edition); however, the metastatic lymph node ratio and 
log odds of positive lymph nodes may be used to better assess prognosis when lymph node sampling was inadequate $(31,32)$. In addition to the status of lymph node metastasis, the factors affecting the prognosis of gastric cancer also included TNM stage, depth of tumor infiltration, vascular invasion and nerve invasion $(33,34)$. Histological classification (34) and tumor size $(35,36)$ may also be used to predict the prognosis of patients with advanced gastric carcinoma. We also evaluated the prognostic factors of patients following gastric cancer surgery, with the single-factor analysis showing that lymph node metastasis, TNM stage and intravascular cancer emboli or perineural invasion were the factors affecting patient survival, while the other factors, including gender, age, tumor site, pathology and different chemotherapies, were not significantly correlated with prognosis. The multivariate Cox regression analysis indicated that lymph node metastasis and TNM stage were independent prognostic factors for the patients (Table V).

In conclusion, our study analyzed 102 cases of gastric carcinoma patients receiving CRT following D2 surgery, with similar overall survival rates observed compared with previous reports, while the toxic side effects were reduced by using IMRT. No significant difference in survival rates was observed between oral capecitabine or intravenous infusion of fluorouracil. However, concurrent chemotherapy with capecitabine was associated with fewer hematological toxicities and gastrointestinal reactions compared with intravenous infusion of fluorouracil; thus, the patients were able to better tolerate and complete the radiotherapy.

\section{Acknowledgements}

The authors would like to thank the colleagues at the Department of Radiation Oncology of Anhui Provincial Hospital for the helpful discussions.

\section{References}

1. Jemal A, Siegel R, Ward E, Hao Y, Xu J and Thun MJ: Cancer statistics, 2009. CA Cancer J Clin 59: 225-249, 2009.

2. Parkin DM, Bray F, Ferlay J and Pisani P: Global cancer statistics, 2002. CA Cancer J Clin 55: 74-108, 2005.

3. Yoo CH, Noh SH, Shin DW, Choi SH and Min JS: Recurrence following curative resection for gastric carcinoma. Br J Surg 87: 236-242, 2000

4. Landry J, Tepper JE, Wood WC, Moulton EO, Koerner F and Sullinger J: Patterns of failure following curative resection of gastric carcinoma. Int J Radiat Oncol Biol Phys 19: 1357-1362, 1990.

5. Lim DH, Kim DY, Kang MK, Kim YI, Kang WK, Park CK, Kim S, Noh JH, Joh JW, Choi SH, et al: Patterns of failure in gastric carcinoma after D2 gastrectomy and chemoradiotherapy: A radiation oncologist's view. Br J Cancer 91: 11-17, 2004.

6. Wisbeck WM, Becher EM and Russell AH: Adenocarcinoma of the stomach: Autopsy observations with therapeutic implications for the radiation oncologist. Radiother Oncol 7: 13-18, 1986.

7. Gunderson LL: Gastric carcer-patterns of relapse after surgical resection. Semin Radiat Oncol 12: 150-161, 2002.

8. Hundahl SA, Philips JL and Menck HR: The National Cancer Data Base Report on poor survival of U.S. gastric carcinoma patients treated with gastrectomy: Fifth edition American Joint Committee on Cancer Staging, proximal disease and the 'different disease' hypothesis. Cancer 88: 921-932, 2000.

9. Kulig J, Kolodziejczyk P, Sierzega M, Bobrzynski L, Jedrys J, Popiela T, Dadan J, Drews M, Jeziorski A, Krawczyk M, et al: Adjuvant chemotherapy with etoposide, adriamycin and cisplatin compared with surgery alone in the treatment of gastric cancer: A phase III randomized, multicenter, clinical trial. Oncology 78: $54-61,2010$.
10. Di Costanzo F, Gasperoni S, Manzione L, Bisagni G, Labianca R, Bravi S, Cortesi E, Carlini P, Bracci R, Tomao S, et al: Adjuvant chemotherapy in completely resected gastric cancer: A randomized phase III trial conducted by GOIRC. J Natl Cancer Inst 100: 388-398, 2008.

11. Macdonald JS, Smalley SR, Benedetti J, Hundahl SA, Estes NC, Stemmermann GN, Haller DG, Ajani JA, Gunderson LL, Jessup JM and Martenson JA: Chemoradiotherapy after surgery compared with surgery alone for adenocarcinoma of the stomach or gastroesophageal junction. N Engl J Med 345: 725-730, 2001.

12. Kim S, Lim DH, Lee J, Kang WK, MacDonald JS, Park CH, Park SH, Lee SH, Kim K, Park JO, et al: An observational study suggesting clinical benefit for adjuvant postoperative chemoradiation in a population of over 500 cases after gastric resection with D2 nodal dissection for adenocarcinoma of the stomach. Int J Radiat Oncol Biol Phys 63: 1279-1285, 2005.

13. Lee J, Limdo H, Kim S, Park SH, Park JO, Park YS, Lim HY, Choi MG, Sohn TS, Noh JH, et al: Phase III trial comparing capecitabine plus cisplatin vs. capecitabine plus cisplatin with concurrent capecitabine radiotherapy in completely resected gastric cancer with D2 lymph node dissection: The ARTIST trial. J Clin Oncol 30: 268-273, 2012.

14. Milano MT, Garofalo MC, Chmura SJ, Farrey K, Rash C, Heimann R and Jani AB: Intensity-modulated radiation therapy in the treatment of gastric cancer: Early clinical outcome and dosimetric comparison with conventional techniques. Br J Radiol 79: 497-503, 2006.

15. Ringash J, Perkins G, Brierley J, Lockwood G, Islam M, Catton P, Cummings B, Kim J, Wong R and Dawson L: IMRT for adjuvant radiation in gastric cancer: A preferred plan? Int J Radiat Oncol Biol Phys 63: 732-738, 2005.

16. Sobin LH, Gospodarowicz MK and Wittekind C (eds): International Union Against Cancer (UICC) TNM Classification of Malignant Tumours. 7th edition. Wiley-Liss, New York, pp73-pp76, 2010.

17. Smalley SR, Gunderson L, Tepper J, Martenson JA Jr, Minsky B, Willett $\mathrm{C}$ and Rich T: Gastric surgical adjuvant radiotherapy consensus report: Rationale and treatment implementation. Int J Radiat Oncol Biol Phys 52: 283-293, 2002.

18. Tepper JE and Gunderson LL: Radiation treatment parameters in the adjuvant postoperative therapy of gastric cancer. Semin Radiat Oncol 12: 187-195, 2002.

19. Trotti A, Colevas AD, Setser A, Rusch V, Jaques D, Budach V, Langer C, Murphy B, Cumberlin R, Coleman CN and Rubin P: CTCAE v3.0: Development of a comprehensive grading system for the adverse effects of cancer treatment. Semin Radiat Oncol 13: 176-181, 2003.

20. Oblak I, Velenik V, Anderluh F and Strojan P: Results of adjuvant radiochemotherapy for gastric adenocarcinoma in Slovenia. EurJ Surg Oncol 33: 982-987, 2007.

21. Macdonald JS, Benedetti J, Smalley S, et al: Chemoradiation of resected gastric cancer: A 10-year follow-up of the phase III trial INT0116 (SWOG 9008). J Clin Oncol 27: 4515-4520, 2009.

22. Boda-Heggemann J, Hofheinz RD, Weiss C, Mennemeyer P, Mai SK, Hermes P, Wertz H, Post S, Massner B, Hieber U, et al: Combined adjuvant radiochemotherapy with IMRT/XELOX improves outcome with low renal toxicity in gastric cancer. Int J Radiat Oncol Biol Phys 75: 1187-1195, 2009.

23. Minn AY, Hsu A, La T, Kunz P, Fisher GA, Ford JM, Norton JA, Visser B, Goodman KA, Koong AC and Chang DT: Comparison of intensity-modulated radiotherapy and 3-dimensional conformal radiotherapy as adjuvant therapy for gastric cancer. Cancer 116: 3943-3952, 2010.

24. Badakhshi H, Gruen A, Graf R, Boehmer D and Budach V: Image-guided intensity-modulated radiotherapy for patients with locally advanced gastric cancer: A clinical feasibility study. Gastric Cancer 17: 537-541, 2014.

25. Vaishampayan UN, Ben-Josef E, Philip PA, Vaitkevicius VK, Du W, Levin KJ and Shields AF: A single-institution experience with concurrent capecitabine and radiation therapy in gastrointestinal malignancies. Int J Radiat Oncol Biol Phys 53: 675- 679, 2002.

26. Osti MF, Agolli L, Bracci S, Monaco F, Tubin S, Minniti G, De Sanctis V and Enrici RM: Adjuvant chemoradiation with 5-fluorouracil or capecitabine in patients with gastric cancer after D2 nodal dissection. Anticancer Res 32: 1397-1402, 2012.

27. Hofheinz RD, Wenz F, Lukan N, Mai S, Kripp M, Staiger W, Schwarzbach M, Willeke F, Möhler M, Post S and Hochhaus A: Oxaliplatin and capecitabine-based chemoradiotherapy for gastric cancer-an extended phase I MARGIT and AIO trial. Int J Radiat Oncol Biol Phys 73: 142-147, 2012. 
28. Bang YJ, Kim YW, Yang HK, Chung HC, Park YK, Lee KH, Lee KW, Kim YH, Noh SI, Cho JY, et al: Adjuvant capecitabine and oxaliplatin for gastric cancer after D2 gastrectomy (CLASSIC): A phase 3 open-label, randomised controlled trial. Lancet 379: 315-321, 2012.

29. Kim DY, Seo KW, Joo JK, Park YK, Ryu SY, Kim HR, Kim YJ and Kim SK: Prognostic factors in patients with node-negative gastric carcinoma: A comparison with node-positive gastric carcinoma. World J Gastroenterol 12: 1182-1186, 2006.

30. Saito H, Kuroda H, Matsunaga T, Fukuda K, Tatebe S, Tsujitani S and Ikeguchi M: Prognostic indicators in node-negative advanced gastric cancer patients. J Surg Oncol 101: 622-625, 2010.

31. Lemmens VE, Dassen AE, van der Wurff AA, Coebergh JW and Bosscha K: Lymph node examination among patients with gastric cancer: Variation between departments of pathology and prognostic impact of lymph node ratio. Eur J Surg Oncol 37: 488-496, 2011.

32. Kim CY and Yang DH: Adjustment of $\mathrm{N}$ stages of gastric cancer by the ratio between the metastatic and examined lymph nodes. Ann Surg Oncol 16: 1868-1874, 2009.
33. Deng J, Liang H, Sun D, Zhang R, Zhan H and Wang X: Prognosis of gastric cancer patients with node-negative metastasis following curative resection: Outcomes of the survival and recurrence. Can J Gastroenterol 22: 835-839, 2008.

34. BaiocchiGL, Tiberio GA, Minicozzi AM, Morgagni P, Marrelli D, Bruno L, Rosa F, Marchet A, Coniglio A, Saragoni L, et al: A multicentric Western analysis of prognostic factors in advanced, node-negative gastric cancer patients. Ann Surg 252: 70-73, 2010.

35. Du C, Zhou Y, Huang K, Zhao G, Fu H and Shi Y: Defining a high-risk subgroup of pathological T2NO gastric cancer by prognostic risk stratification for adjuvant therapy. J Gastrointest Surg 15: 2153-2158, 2011.

36. Qiu MZ, Wang ZQ, Luo HY, Zhang DS, Zhou ZW, Li YH, Jiang WQ and Xu RH: Prognostic analysis in node-negative gastric cancer patients in China. Tumor Biol 32: 489-492, 2011. 